\title{
Measuring the morphology and density of internally mixed black carbon with SP2 and VTDMA: new insight into the absorption enhancement of black carbon in the atmosphere
}

Yuxuan Zhang et al.

Correspondence to: Qiang Zhang (qiangzhang@tsinghua.edu.cn) and Hang Su (h.su@mpic.de)

The copyright of individual parts of the supplement might differ from the CC-BY 3.0 licence. 
For ambient BC-free and BC-containing particles, the optical diameters at peaks of number size distribution derived from SP2 measurement were chosen to compare with the prescribed mobility size (through DMA), shown in Fig. S1. The difference between optical size and mobility size was $3 \%$ and $0.3 \%$ for ambient BC-free particles and BCcontaining particles, respectively. The excellent coherence demonstrated the validity of LEO method for ambient measurements.

In our study, two types of ambient BC particles was divided based on the delay time distribution, shown in Fig. S2. The observed two peaks of delay time distribution is due to the difference in behavior of Ex-BC and In-BC particles during SP2 measurement. The first peak with lower delay time represents the Ex-BC particles. Corresponding, the second peak is considered as the proxy of In-BC particles. Therefore, the particles with delay time more than $1.6 \mu$ s was chose to investigate their core morphology and density.

The $R I_{\text {nonBC }}$ was determined by combining SP2 and VTDMA measurements in our study. In previous SP2 studies, the $R I_{\text {nonBC }}$ is usually given an assumption value of around 1.50. The $R I_{\text {nonBC }}$ shown in Figure $\mathrm{S} 3$ was calculated using the scattering properties from SP2 measurement and particle sizes from DMA1, respectively. The $R I_{\text {nonBC }}$ distribution ranged from 1.2 to 1.8 for single charged non-BC particles at 200$350 \mathrm{~nm}$. We selected the peak value of 1.42 as the $R I_{\text {nonBC }}$ for Mie theory calculations. 

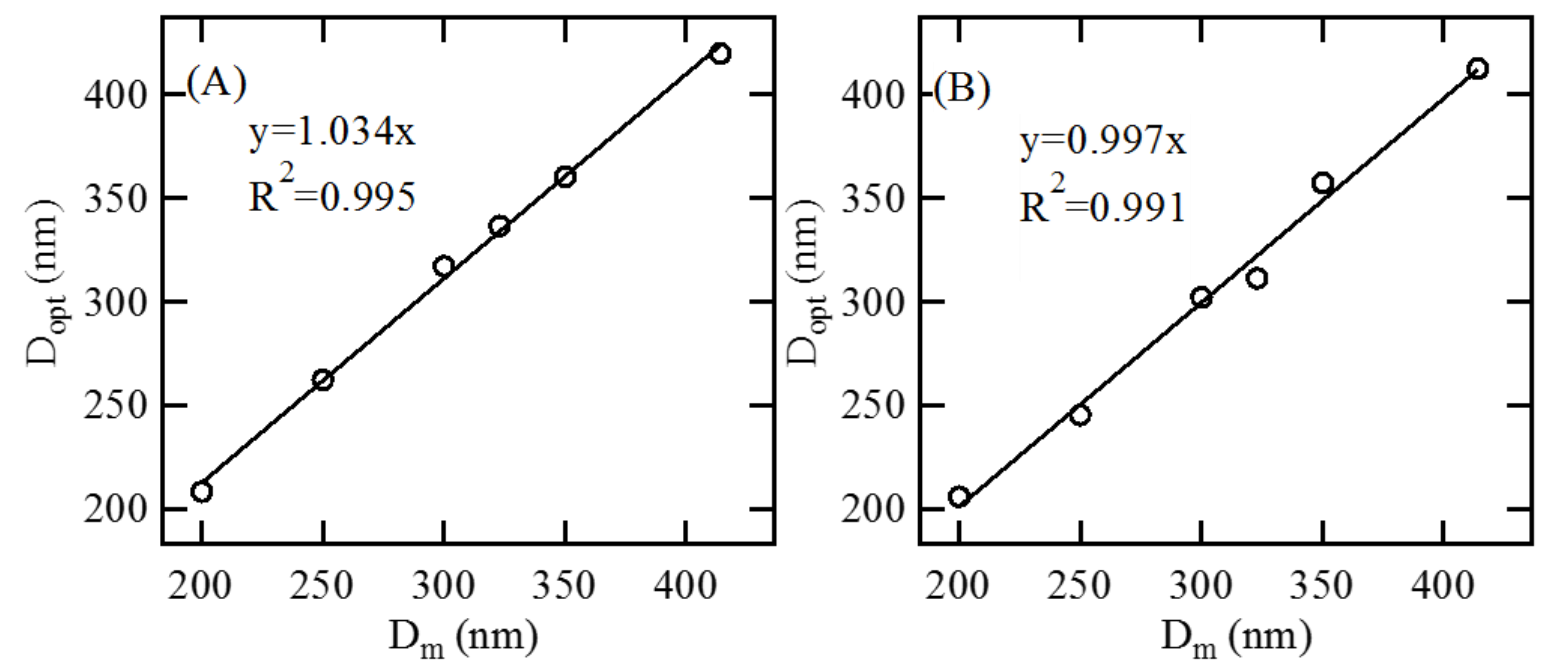

1

2 Figure S1. Mobility size vs. optical size for ambient BC-free particles (A) and BC-

3 containing particles (B) observed in Xianghe site. 


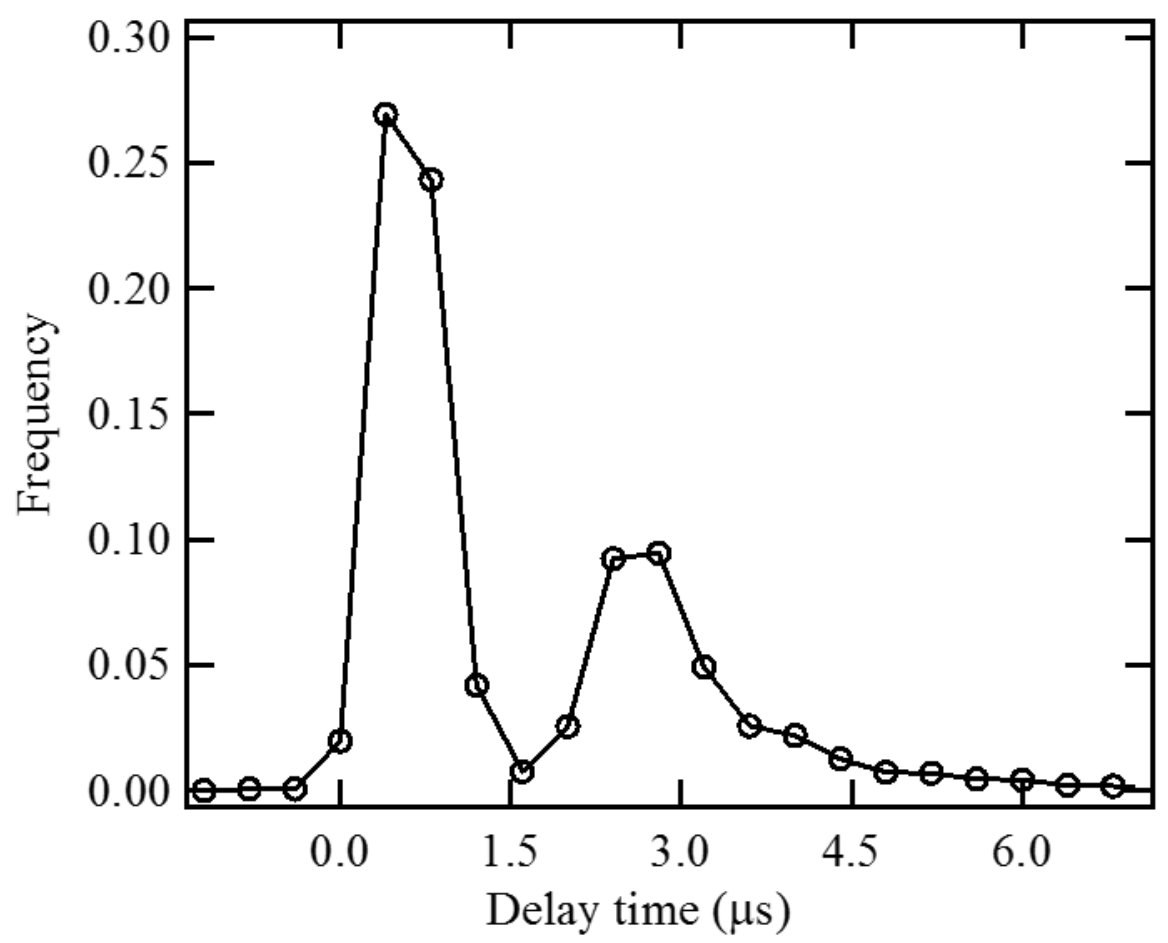

2

3 Fig. S2. The distribution of delay time in our SP2 measurement. 


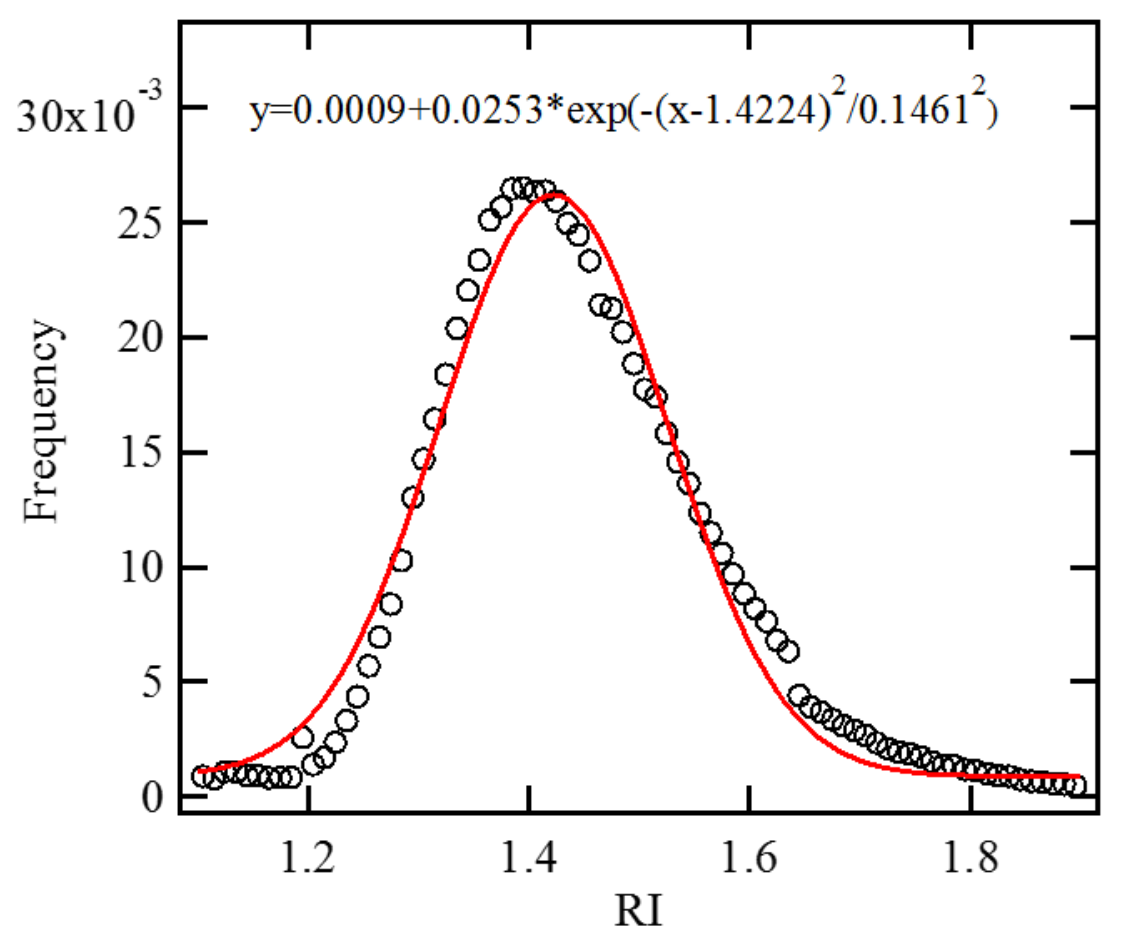

Fig. S3. The RI distribution for non-BC particles. 

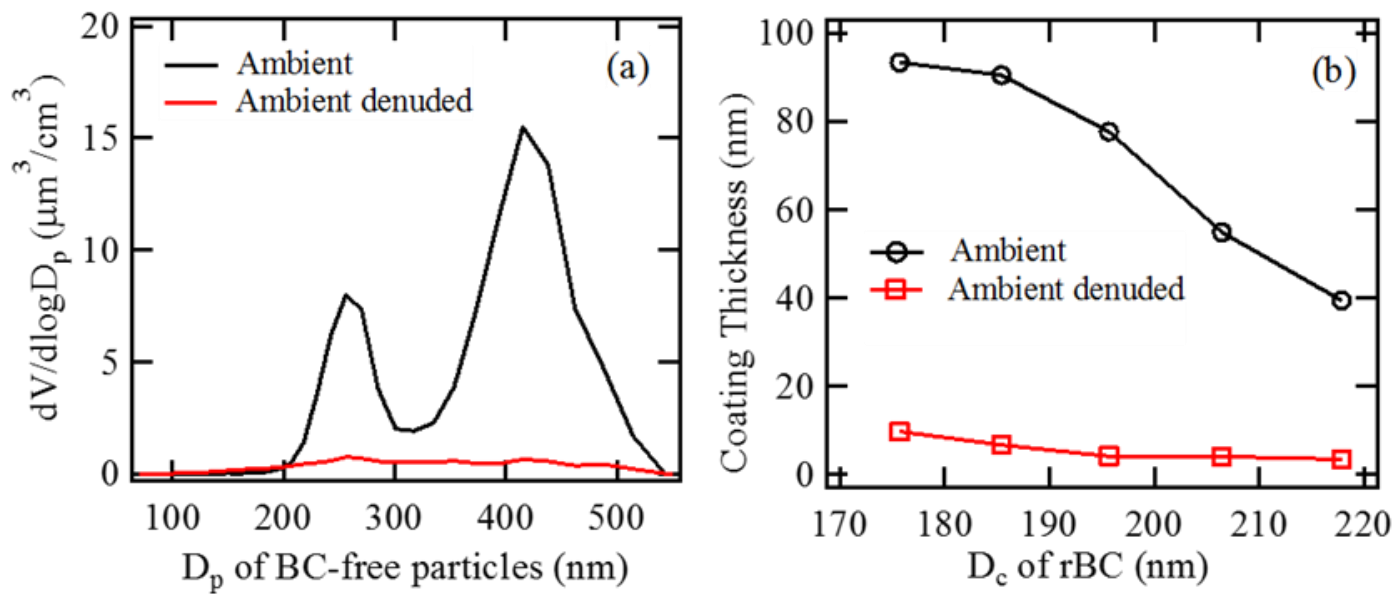

Figure S4. The removal of non-refractory component for ambient BC-free and BCcontaining particles using a thermo-denuder at $300{ }^{\circ} \mathrm{C}$ : (a) the volume size distribution of ambient BC-free particles before and after heating, (b) coating thickness of sizeresolved $\mathrm{rBC}$. The ambient particles was selected by the DMA1 prescribed $250 \mathrm{~nm}$. 